\title{
Structure-Property Investigations via SEM In-Situ Micromechanical Testing
}

\author{
Robert Wheeler ${ }^{1}$, Dhriti Bhattacharya ${ }^{2}$, Amit Pandey ${ }^{3,4}$, Amit Shyam ${ }^{3}$, Adam Shiveley ${ }^{5}$ and Daniel \\ Sergison $^{6}$ \\ ${ }^{1}$ MicroTesting Solutions LLC, Hilliard, OH, USA \\ 2 Australian Nuclear Science and Technology Organization, Lucas Heights, Australia \\ ${ }^{3}$ Materials Science and Technology Division, Oak Ridge National Laboratory, Oak Ridge, TN, USA \\ ${ }^{4}$ LG Fuel Cell Systems Inc., North Canton, OH, USA \\ ${ }^{5}$ Shiveley Technology Corp., Dayton, OH, USA \\ ${ }^{6}$ Sergison Machine, East Peoria, IL, USA
}

Small-scale in-situ testing within the SEM can be used to study deformation behavior in materials, providing some of the most compelling insights into mechanisms that govern mechanical properties. In-situ micro- and nanomechanical tests conducted on well-shaped specimens provide information about a full suite of properties of interest (e.g. yield phenomena, strength, ductility, modulus, work hardening, etc.). This occurs while simultaneously affording a glimpse into the dynamic structural changes that reflect underlying dislocation processes associated with those properties (e.g. local versus global slip activity, twinning, grain boundary interactions, slip about second phases, necking, etc.). The microstructural evidence can be in the form of secondary and backscattered electron images, ion images or in orientation determination from EBSD analyses. The power of the SEM in-situ microscale test methodology is that it encompasses analysis of the entire body being deformed with the resolution of key features responsible for the structure-property correlation [1]. And further, it inherently provides the ability to study time-dependent development of microstructure during deformation. This has profound implications for deformation modeling where simulation over the entire test volume has now become a tractable endeavor.

Results from different studies will be presented to illustrate the relationship between the stress-strain response of metals and the associated change in microstructure as plastic flow proceeds. In one case, single crystal iron foil microsamples were deformed in tension under quasi-static loading conditions. Specimens were machined in the FIB such that the longitudinal direction of the samples, parallel to the loading axis, was along the $<110>$ direction. Following small strain increments, the crosshead displacement was arrested and images of the deforming specimens were recorded. Periodically, the rectangular samples were analyzed by EBSD to investigate the occurrence of crystal rotation. Figure 1(a) and (b) shows the microtension specimen at two different levels of deformation relative to the stress strain curve in Figure 1(c). Crystal rotations have been identified in macroscopic specimens for similarly loaded single crystals of iron near the fracture surface [2]. The IPF and $\{111\}$ pole figure analysis in Figure 1(d) confirms local crystal rotations resulting from slip on inclined $<111>\{112\}$ slip systems.

In a separate study, tension tests were carried out on polycrystalline $\mathrm{Cu}$ samples having different grain sizes. The stress-time plot in Figure 2 shows the loading and subsequent hold response of a sample with grain size of approximately $2 \mathrm{um}$. In these quasi-static loading tests, stress-strain data at the microscopic scale suffer from the same variation with time that is found in larger test specimens [3]. The inset of Figure 2 shows the transition from elastic to plastic strain near yielding. Stress relaxation (SR) is indicated by the load drop seen in the hold stages of the test, while SEM images are acquired. This phenomenon, associated with irreversible dislocation motion, is manifested in the images as well. 
Quantitative strain evidence contained in these images represents a graded range as measured from top to bottom of the serially acquired images [3]. Unique insights into SR are afforded by the full volume analysis presented by in-situ micromechanical testing.

The case studies presented here will illustrate how certain SEM in-situ micromechanical tests can be used to enable experimental setups. We discuss how these tests can be designed to integrate appropriate test modes with the specimen size and shape required to address a specific behaviour or property.

\section{References:}

[1] R. Wheeler, P. A. Shade and M. D. Uchic, JOM 64(1) (2012), p.58-65.

[2] R. N. Gardner, and H. G. F. Wilsdorf, Metallurgical Transactions A 11 (1980), p. 659-669.

[3] A. D. Kammers and S. Daly, Experimental Mechanics 53.9 (2013), p. 1743-1761.
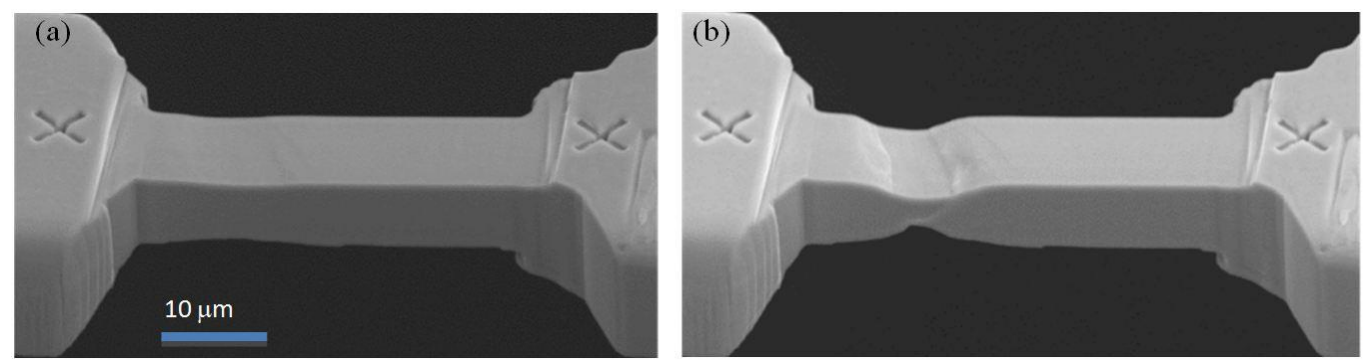

(C)
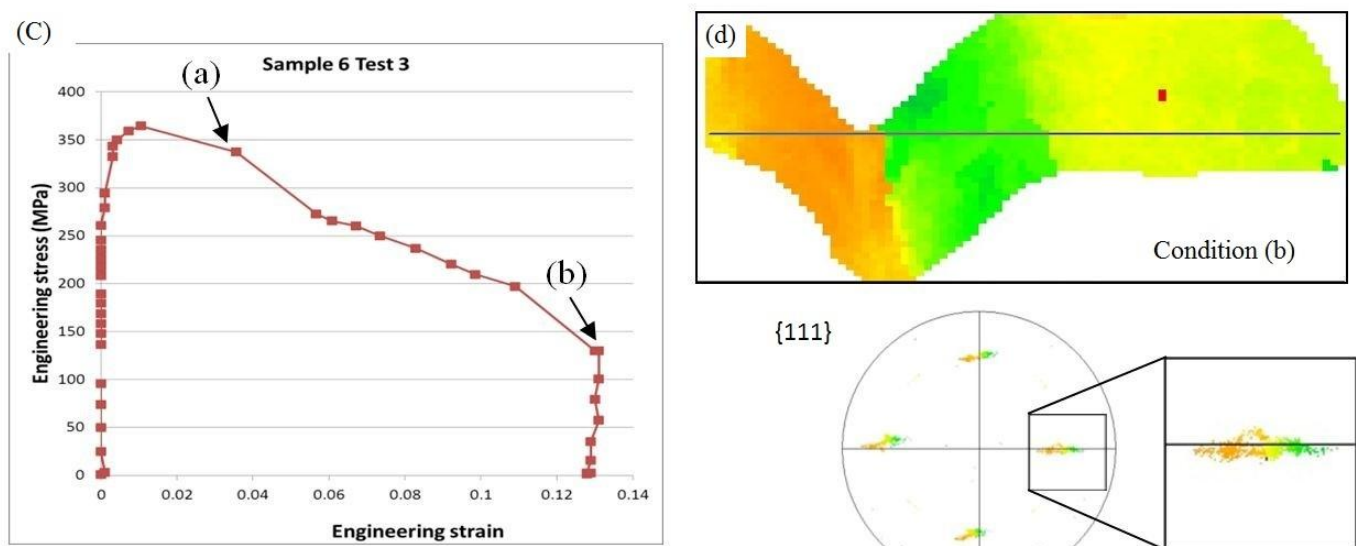

$\{111\}$

Figure 1. Iron single crystal microtension sample, (a) image after yielding and (b) after 12\% strain, (c) stress strain plot, and (d) EBSD analysis at $12 \%$ strain.

Figure 2. Stress-time data for a microtension test on a fine-grained copper sample with grain size of 2 microns. Inset shows transition from elastic to plastic behavior where stress relaxation is observed during the image hold stages (horizontal sections) once plastic flow is initiated.

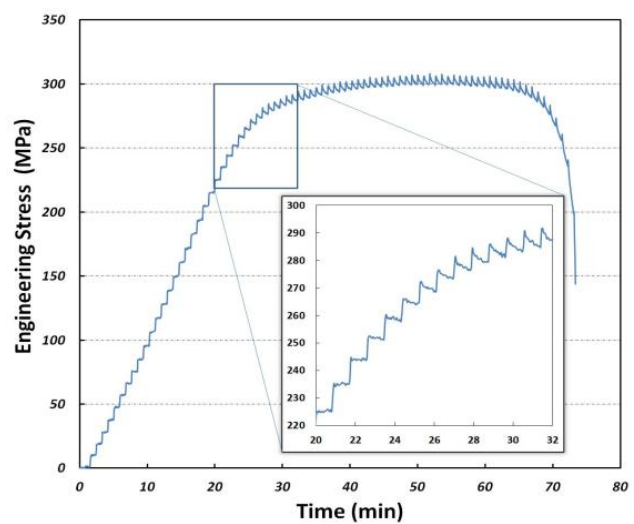

